\title{
Plataforma Moodle para desarrollar el pensamiento crítico y creativo en educación primaria
}

\author{
Mg. María Cecilia Delgado Orrillo \\ dorrillomc@ucvvirtual.edu.pe \\ https://orcid.org/0000-0003-1521-3175
}

Mg. Lina Silvia Chávez Quinteros

cquinterosls@ucvvirtual.edu.pe https://orcid.org/0000-0001-6021-1991

Programa Académico de Doctorado en Educación

Escuela de postgrado Universidad César Vallejo

Chiclayo - Perú

\section{RESUMEN}

La presente investigación tuvo como objetivo demostrar la influencia que genera el uso de la plataforma Moodle en el desarrollo del pensamiento crítico y creativo en los estudiantes del sexto grado de la Institución Educativa $N^{\circ} 10384$ en la provincia de Chota -Cajamarca, para lo cual se realizó una investigación de tipo aplicada y enfoque cuantitativo, el diseño utilizado fue experimental en su nivel cuasi experimental con dos grupos. En dicho estudio, participaron 34 estudiantes en el grupo experimental y 35 estudiantes en el grupo control. Se aplicó un test validado por juicio de 05 expertos, que permitió recoger información mediante 20 ítems sobre el pensamiento crítico y creativo. Según los resultados que arrojó el pre test la mayoría de estudiantes se encontraban en el nivel inicio, luego de aplicar el Programa de Experiencias de Aprendizaje utilizando la plataforma virtual Moodle, los resultados del post test evidencian el desarrollo del pensamiento crítico y creativo en los estudiantes del grupo experimental a diferencia del grupo control cuyos resultados demuestran que no hubo avance. Por lo tanto, se concluye que el uso de la Plataforma Moodle influye significativamente en el desarrollo del pensamiento crítico y creativo.

Palabras clave: pensamiento crítico y creativo; programa, plataforma virtual moodle. 


\title{
Moodle platform to develop critical and creative thinking in primary education
}

\begin{abstract}
The present research aimed to demonstrate the influence generated by the use of the Moodle platform in the development of critical and creative thinking in sixth grade students of the Educational Institution No. 10384 in the province of Chota-Cajamarca, the type of research was applied and with a quantitative methodological approach, the design used was experimental at its quasi-experimental level with a control group with 35 students and an experimental group with 34 students. A test validated by 05 experts was applied, which allowed collecting information through 20 items, according to dimensions of critical and creative thinking. The results indicated that the majority of students in both groups were at the beginning level and after participating in the learning experiences program using the virtual moodle platform, the results show that the development of critical and creative thinking rose in the students. students of the experimental group what did not happen in the control group. Concluding that the use of the moodle virtual platform significantly influences the development of critical and creative thinking.
\end{abstract}

Keywords: critical and creative thinking; program, virtual platform moodle.

Artículo recibido: 30 noviembre. 2021 Aceptado para publicación: 29 diciembre 2021 Correspondencia: dorrillomc@ucvvirtual.edu.pe Conflictos de Interés: Ninguna que declarar 


\section{INTRODUCCIÓN}

Actualmente la educación es un proceso que se adapta a las necesidades e intereses de nuestra sociedad, es `por ello que se toma el modelo educativo por competencias en donde el estudiante debe lograr aprendizajes que le permitan lograr un mejor desempeño en su entorno social y personal. Siendo el pensamiento crítico y creativo una de las habilidades de orden superior y la tecnología un aspecto indispensable en el proceso educativo.

Actualmente la educación es un proceso que se adapta a las necesidades e intereses de nuestra sociedad, es por ello que se toma el modelo educativo por competencias en donde el estudiante debe conseguir aprendizajes que le permitan lograr un mejor desempeño en el entorno social y personal. Dicho esto, la tarea docente se centra en una formación basada no sólo en contenidos sino en la aplicación de éstos en un contexto real pensando de manera crítica y creativa, situación que se constituye en un tema preponderante en todos los niveles de educación. Para lo cual es necesario que los docentes profundicemos nuestro conocimiento acerca del tema, así como ir investigando y aplicando diversas estrategias metodológicas que lo fortalezcan.

En Ginebra-Suiza se realizó un festival educativo sobre pensamiento crítico, en enero de 2020, incidiendo en la relevancia de cuestionar información recibida, analizar contenidos, expresar un punto de vista personal fomentando de esta manera el pensamiento crítico en actividades cotidianas, tanto personales como sociales (Bachillerato Internacional, 2020). Según Alvino (2019) en Veracruz - México se realizó el IV Seminario Internacional de pensamiento crítico, siendo considerado como uno de los retos de la educación en un contexto tan cambiante que requiere de permanente actualización pedagógica para lograr una formación integral de nuestros estudiantes en conocimientos, habilidades y actitudes, así como un pensamiento reflexivo, crítico y creativo y desarrollo de la autonomía.

En Lima se llevó a cabo el I Torneo Internacional de debate Interescolar "Expresarte América 2018" participando los finalistas de diferentes regiones como Piura, La Libertad, Cusco, San Martín, Lima y Junín para debatir temas de actualidad, destacando que, estos eventos fomentan el pensamiento crítico en medio de una competencia sana, de respeto y tolerancia (Congreso de la Repúlica Perú, 2018).

El Proyecto Educativo Local de la UGEL Hualgayoc - Bambamarca, 2017 -2021, con el objetivo de lograr aprendizajes significativos y mejorar la calidad educativa de su medio, considera como una de las acciones estratégicas para lograr sus objetivos trazados fortalecer 
el pensamiento crítico, creativo y valorativo en los educandos, por ser consideradas habilidades muy necesarias a desarrollar en la actualidad (Unidad de Gestión educativa Hualgayoc - Bambamarca, 2017).

La presente investigación se realizó en la Institución Educativa Primaria No 10384, ubicada en el distrito y provincia de Chota - Cajamarca, la institución cuenta con 680 estudiantes matriculados y 18 docentes, tres secciones de Sexto Grado "A", "B" y "C"; conformadas por 35, 34 y 33 estudiantes respectivamente. Se observó que los estudiantes tienen deficiencias en el desarrollo del pensamiento crítico y creativo, con manifestaciones como las limitaciones para interpretar, comprender y mantener un juicio crítico de lo que leen o escuchan, desarrollo de trabajos poco originales, los estudiantes reciben pasivamente información que ofrece el docente y rara vez cuestionan, no asumen autonomía ante la toma de decisiones en situaciones cotidianas y mayormente se dejan llevar por otros. Sin embargo, en la escuela se siguen realizando actividades y experiencias repetitivas y descontextualizadas, no se aplican estrategias didácticas para fortalecer un pensamiento crítico y creativo.

Entre las teorías en las que se fundamenta la investigación se tiene a la Constructivista de Piaget. El desarrollo de estructuras cognitivas se dan en el proceso de maduración biológica del estudiante, en relación con el contexto en que se desarrolla y su aprendizaje. Para que suceda esto, tiene que pasar por los procesos de asimilación que se relaciona con el contacto que existe entre el individuo y objetos externos, interpreta de acuerdo a sus esquemas mentales adquiridos previamente, generando así sus propios significados. La acomodación hace referencia a como los conceptos ya asimilados se integran a la red cognitiva del individuo, formando estructuras nuevas de pensamiento e ideas relacionadas a su realidad objetiva. Posteriormente aparece el equilibrio, el desequilibrio y el reequilibrio que dan lugar al conocimiento después de haber solucionado el conflicto cognitivo (Arévalo \& Nauta, 2012).

El Modelo de Villarini parte de la perspectiva materialista histórica y de su formulación psicológica histórico - cultural de Lev Vygotsky y sostiene que el pensamiento como una capacidad humana, siempre se encuentra en proceso de desarrollo, es decir la manera cómo piensa el estudiante, está supeditado al contexto en el que vive y de acuerdo a sus necesidades, intereses que pueda experimentar en el contexto, el pensamiento no se desarrolla ajeno a la realidad en que se vive, a él también se integran los diversos 
aprendizaje que pueda adquirir y surgen nuevas funciones psicológicas que puedan suscitar una transformación, lo que lo diferencia del mero conductismo (Villarini, 2019) La teoría de la conectividad posee ocho principios que son: El aprendizaje y el conocimiento se fundamentan en la variedad de opiniones, el aprendizaje es un proceso de conexión de nodos o también llamados fuentes de información especializadas, el aprendizaje puede darse también en dispositivos no humanos, la capacidad de saber más es más crítica que lo actualmente conocidos, para facilitar el aprendizaje continuo es necesario nutrir y mantener las conexiones, la habilidad para mantener las conexiones entre áreas ideas y conceptos es clave, lo vigente el conocimiento actualizado y preciso constituye el proceso de todas las actividades del proceso conectivista, la toma de decisiones es considerada un proceso de aprendizaje. El acto de que el estudiante escoja que aprender, así como el significado de la información que recibe es visto a través de una realidad que está en constante cambio (Velásquez et al., 2021)

Para (Horn \& Veermans, 2019) es muy relevante en la educación básica la aplicación de estrategias que desarrollen el pensamiento crítico, las mismas que lo llevarán a desarrollar un discurso razonado, que hoy en día se considera una de los más grandes logros que la educación pueda alcanzar, habiéndose determinado en varios estudios que a los niños les emociona mucho el saber el cómo, el porqué y el para qué de las cosas.

Según Facione (2017) afirma que el pensamiento creativo, nos conlleva a encontrar algo novedoso, enfoques nuevos, genuinas perspectivas y a comprender de manera muy diferente la concepción de las cosas. Considera como producto de este tipo de pensamiento la danza, la música, la poesía, la literatura dramática, los invento y las innovaciones técnicas. Así también existen otros ejemplos básicos como el hacer una interrogante hacia una posible solución, que nos lleva a concebir el mundo de manera imaginativa y distinta.

Desarrollar el pensamiento creativo desde la familia es una gran alternativa, por la tanto la interacción entre escuela y familia debe ser muy activa dotando al estudiante de diverasas actividades que permitan despertar el interés y la creatividad, las mismas que deben estar plasmadas en la planificación de los docentes haciendo uso de diferentes estrategias que lo conduzcan a tal fin (Unger \& BenDavid-Hadar, 2021). 
En base a diversas investigaciones, se afirma un desarrollo significativo del pensamiento creativo, desde temprana edad de los niños, lo cual tiene una relación con la realización de actividades y juegos tanto en campo abierto como en el aula, afrontando nuevos retos, resolviendo nuevos problemas y actividades que los haga pensar en diversas soluciones ante una misma situación, donde el niño piense de manera creativa y descubra nuevas experiencias (Wojciehowski \& Ernst, 2018)

Para Espinoza \& Zambrano (2020) la Plataforma Moodle es una herramienta virtual que favorece al trabajo compartido que permite el desarrollo de capacidades como las de crear, compartir y transformar el conocimiento, debido a que está basado en un enfoque pedagógico constructivista. Por lo tanto el uso de esta herramienta virtual tiene mucha implicancia en el desarrollo del pensamiento crítico y creativo.

El uso de la plataforma Moodle, permite a los educandos trabajar en aulas virtuales, tener acceso a diversos recursos y materiales de aprendizaje, dando óptimos resultados, debidoa a que a que está diseñado para el trabajo cooperativo y la interacción entre estudiantes de manera sincrónica o asincrónica (Sirwan et al, 2021)

El contexto actual, exige que el enfoque educativo replantee el proceso enseñanza aprendizaje y desde el punto de vista tecnológico darle prioridad al uso de las plataformas virtuales como herramienta didáctica, las mismas que favorecen al trabajo autónomo, la motivación e interación entre estudiantes y estudiante-docente (Guzzetti, 2020)

Algunos líderes de opinión consideran que existen un serie de habilidades del siglo XXI denominándolas las 4C (Pensamiento crítico y resolución de problemas, comunicación, colaboración, creatividad e innovación), dentro de las cuales el pensamiento crítico es una de las habilidades primordiales a desarrollar en la actualidad, debido a que incluyen habilidades como analizar, evaluar y sintetizar más aún las relacionamos con la era digital, en la que el estudiante debe analizar y discernir la abundante información que está a su alcance en la actualidad (Ikhsan et al., 2020).

Las nuevas tecnologías cumplen sin duda un papel muy importante dentro del aprendizaje siendo de gran soporte, para los docentes porque ofrece muchas herramientas que fortalecen su labor pedagógica y a los estudientes porque les brinda la oportunidad de construir su propio aprendizaje, en eeste caso haciendo uso de las plataformas virtuales (De medio et al, 2019). 


\section{ESTRATEGIAS METODOLÓGÍCAS}

\subsection{Tipo y diseño de investigación}

\subsubsection{Tipo de investigación: Aplicada}

El tipo de investigación que se utilizó fue aplicada, debido a que busca generar conocimientos a través de la aplicación de un Programa utilizando la plataforma moodle, orientado al desarrollo del pensamiento crítico y creativo; al respecto Rus (2021) señala que la investigación aplicada está orientada a dar solución a problemas de la sociedad apoyándose en la investigación básica para conseguirlo.

La investigación es de enfoque cuantitativo porque se recogiron y procesaron datos cuantitativos a fin de probar una hipótesis. El enfoque cuantitativo utiliza métodos estadísticos para finalmente extraer conclusiones y probar teorías (Hernández, Fernández y Baptista, 2014) El diseño de investigación es el experimental en su nivel cuasi experimental, cuyo diagrama es el siguiente:

\section{GE: $01 \quad \mathrm{X} \quad 02$ \\ GC: $03 \quad-04$}

\section{Dónde:}

GE: Grupo experimental

GC: Grupo de control

O1: Test sobre pensamiento crítico y creativo aplicado a los estudiantes

X: Aplicación del Programa de Experiencias de Aprendizaje utilizando la plataforma virtual moodle con los estudiantes

O2: Test sobre pensamiento crítico y creativo aplicado a los estudiantes del sexto grado que conforman el grupo experimental, después de la aplicación del programa de experiencias de aprendizaje utilizando la plataforma moodle

O3: Pre test sobre pensamiento crítico y creativo aplicado a los estudiantes de sexto grado que conforman el grupo de control.

O4: Pos Test sobre pensamiento crítico y creativo aplicado a los estudiantes de sexto grado que conforman el grupo de control.

\subsection{Variables y operacionalización}

\subsubsection{Variable 1 y/o independiente: Plataforma virtual Moodle}

2.2.2. Variable 2 y/o dependiente: Pensamiento crítico y creativo.

2.2.3. La operacionalización de las variables se encuentra ubicada en el anexo 01. 


\subsection{Población, muestra, muestreo}

\subsubsection{Población}

La población estuvo conformada por 102 estudiantes del sexto grado de educación primaria de la Institución Educativa (I.E.) N 10384 - Chota; para Robles (2019) la población es considerada como el conjunto de personas, objetos, transacciones o eventos en los que se está interesado en estudiar.

\section{Tabla 1}

Distribución del número de estudiantes del sexto grado de la I.E. N 10384 Chota Cajamarca 2021.

\begin{tabular}{cccc}
\hline SECCION & VARONES & MUJERES & TOTAL \\
\hline A & 13 & 22 & 35 \\
B & 19 & 15 & 34 \\
C & 18 & 15 & 33 \\
\hline TOTAL & $\mathbf{5 0}$ & $\mathbf{5 2}$ & $\mathbf{1 0 2}$ \\
\hline
\end{tabular}

Nota: Nominas de matrícula 2021

La población estuvo conformada por 102 estudiantes del sexto grado de educación primaria de la Institución Educativa (I.E.) N 10384 - Chota; para Robles (2019) concibe a la población como la agrupación de personas, objetos, transacciones o eventos en los que se está interesado en estudiar.

\subsubsection{Muestra}

La muestra para la presente investigación se realizó utilizando la técnica probabilística del azar simple a nivel de secciones, grupos constituidos, determinando así un grupo experimental con 34 estudiantes, el mismo que participó en el proceso de aplicación del programa de experiencias de aprendizaje utilizando la plataforma virtual Moodle y un grupo de control con 35 estudiantes, que no participó del experimento.

\section{Tabla 2}

Muestra de estudio constituida por 69 que conformaron los dos grupos de estudio: grupo experimental y control

\begin{tabular}{lccc}
\multicolumn{1}{c}{ Grupos } & VARONES & MUJERES & TOTAL \\
\hline Grupo Experimental & 13 & 22 & 35 \\
Grupo Control & 19 & 15 & 34 \\
\hline TOTAL & 32 & 37 & 69 \\
\hline
\end{tabular}

Nota: Nominas de matrícula 2021 


\subsection{Técnicas e instrumentos de recolección de datos}

En la presente investigación se utilizó la técnica de la observación a través de una prueba de conocimientos o test, aplicado antes y después del experimento tanto al grupo experimental como de control con la finalidad de recoger información relacionada con el desarrollo del pensamiento crítico y creativo. Para Herrera (2020) la técnica de la observación va más allá de abrir los ojos, haciendo referencia a detenerse con atención y minuciocidad ante una información determinada y sus elementos que nos acercan de la manera más concreta a la realidad. Los Test son instrumentos de medida muy usados por profesionales de psicología e investigadores, con la finalidad de obtener muestras de comportamiento de las personas en diversos contextos (Muñiz \& Fonseca-Pedrero, 2019)

\section{RESULTADOS}

Los resultados de esta investigación demiuestran que se logró elebar el pensamiento crítico y creativo de los estudiantes de sexto grado, como se evidencia en las siguientes tablas: Niveles del pensamiento crítico y creativo del grupo experimental y control antes de la plataforma Moodle

Tabla cruzada GRUPO *Pensamiento crítico y creativo pre test

\begin{tabular}{|c|c|c|c|c|c|c|}
\hline & \multicolumn{3}{|c|}{$\begin{array}{c}\text { Pensamiento crítico y creativo } \\
\text { pre test }\end{array}$} & \multirow[t]{2}{*}{ Total } \\
\hline & & & 1 Inicio & 2 Proceso & 3 Logro & \\
\hline \multirow{4}{*}{ GRUPO } & \multirow{2}{*}{ Experimental } & Recuento & 11 & 21 & 2 & 34 \\
\hline & & $\%$ dentro de GRUPO & $32,4 \%$ & $61,8 \%$ & $5,9 \%$ & $100,0 \%$ \\
\hline & \multirow{2}{*}{ Control } & Recuento & 13 & 17 & 5 & 35 \\
\hline & & $\%$ dentro de GRUPO & $37,1 \%$ & $48,6 \%$ & $14,3 \%$ & $100,0 \%$ \\
\hline \multirow{2}{*}{ Total } & & Recuento & 24 & 38 & 7 & 69 \\
\hline & & $\%$ dentro de GRUPO & $34,8 \%$ & $55,1 \%$ & $10,1 \%$ & $100,0 \%$ \\
\hline
\end{tabular}

Nota: Pre test aplicado al grupo experimental y grupo control en pensamiento crítico y creativo.

Se observa que la mayoría de estudiantes en el pre test, se encuentran en el nivel proceso en lo que concierne al desarrollo del pensamiento crítico y creativo. 
Tabla cruzada GRUPO *Pensamiento crítico y creativo post test

\begin{tabular}{|c|c|c|c|c|c|c|c|}
\hline & \multicolumn{4}{|c|}{$\begin{array}{c}\text { Pensamiento crítico y creativo post } \\
\text { test }\end{array}$} & \multirow[t]{2}{*}{ Total } \\
\hline & & & $\begin{array}{c}1 \\
\text { Inicio }\end{array}$ & $\begin{array}{c}2 \\
\text { Proceso }\end{array}$ & $\begin{array}{c}3 \\
\text { Logro }\end{array}$ & $\begin{array}{c}4 \text { Logro } \\
\text { destacado }\end{array}$ & \\
\hline \multirow{4}{*}{ GRUPO } & \multirow{2}{*}{ Experimental } & Recuento & 0 & 1 & 13 & 20 & 34 \\
\hline & & $\%$ dentro de GRUPO & $0,0 \%$ & $2,9 \%$ & $38,2 \%$ & $58,8 \%$ & $100,0 \%$ \\
\hline & \multirow{2}{*}{ Control } & Recuento & 6 & 22 & 7 & 0 & 35 \\
\hline & & $\%$ dentro de GRUPO & $17,1 \%$ & $62,9 \%$ & $20,0 \%$ & $0,0 \%$ & $100,0 \%$ \\
\hline \multirow{2}{*}{ Total } & & Recuento & 6 & 23 & 20 & 20 & 69 \\
\hline & & $\%$ dentro de GRUPO & $8,7 \%$ & $33,3 \%$ & $29,0 \%$ & $29,0 \%$ & $100,0 \%$ \\
\hline
\end{tabular}

Nota: Post test aplicado al grupo experimental y grupo control

Se deduce que la mayoría de estudiantes en el post test, se encuentran en el nivel logro destacado en lo que concierne al desarrollo del pensamiento crítico y creativo, evidenciándose que la aplicación del programa de experiencias de aprendizaje utilizando la Plataforma virtual Moodle, ha incrementado de manera muy significativa el nivel de logro de los estudiantes.

\section{DISCUSIONES}

En la presente investigación se tuvo como objetivo demostrar la influencia que genera el uso de la plataforma virtual moodle en el desarrollo del pensamiento crítico y creativo en

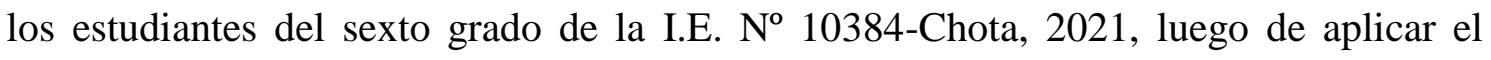
programa de experiencias de aprendizaje con la plataforma moodle, se obtuvo como resultado que los estudiantes lograron elevar el nivel de pensamiento crítico y creativo en comparación al nivel que obtuvieron antes de la aplicación del programa en mención, los indicadores que se abordaron tienen como antecedente a la investigación realizada en Colombia de

La propuesta pedagógica presentada, tuvo fundamentación teórica en la teoría constructivista de Piaget, quien concibe al estudiante como un individuo activo de su mismo aprendizaje, quien construye sus propios conocimientos significativamente utilizando procesos de selección, organización, transferencia y recuperación, contando con la orientación del docente (Arévalo \& Nauta, 2020). El programa de experiencias de aprendizaje aplicado con la plataforma virtual moodle, se basa en esta teoría por ser de carácter constructivista en la que los estudiantes desarrollaron actividades que 
permitieron que sean ellos mismos los que construyan sus aprendizajes ya sea de, manera sincrónica o asincrónica.

Otra de los fundamentos teóricos de la investigación es el Modelo de Villarini, quien a partir de la teoría de Vigotski sostiene que, el pensamiento es una capacidad humana que se encuentra siempre en proceso de desarrollo relacionado estrechamente con el contexto en el cual se desenvuelve Villarini (2019). Esta teoría se relaciona con la investigación realizada porque el uso de la plataforma moodle permitió realizar con los estudiantes un trabajo colaborativo, donde se interactúa con los estudiantes intercambiando ideas, desarrollando trabajos grupales, foros de discusión, favoreciendo al desarrollo del pensamiento crítico y creativo.

Otra de las teorías en la cual está fundamentada esta investigación, es la teoría del Conectividad, del autor canadiense George Siemens, teoría se desarrollada en el 2005, caracterizada por estar inmersa en el mundo socio - digital, en la creación de redes y conexión de nodos, además de conexiones conceptuales y sociales, hacia el logro de aprendizajes significativos (Velásquez et al., 2021)

La propuesta pedagógica consistió en diseñar y aplicar un programa de experiencias de aprendizaje, plasmadas en la plataforma virtual moodle utilizando los recursos y actividades que ofrece esta plataforma, siendo los más usados los foros de debate y discusión, las etiquetas, los recursos interactivo como los vídeos y los cuestionarios.

Las dificultades que se presentaron para el desarrollo de este trabajo radican en el uso de dispositivos digitales, ya que a pesar de que todos los niños cuentan con tabletas para realizar el trabajo, la zona en que se desarrolló no tiene una cobertura eficiente, interrumpiendo en varias ocasiones las actividades programadas con los estudiantes.

\section{CONCLUSIONES}

Se diagnosticó el nivel de pensamiento crítico y creativo en los estudiantes de la Institución Educativa $\mathrm{N}^{\mathrm{o}} 10384$ de la provincia de Chota, en donde la mayoría de los estudiantes se ubican en el nivel inicio y proceso en la variable general, así como en cada una de sus dimensiones. Se diseñó y aplicó el Programa de experiencias de aprendizaje en la plataforma virtual Moodle como estrategia `para desarrollar el pensamiento crítico y creativo de los estudiantes del sexto grado de la Institución educativa $\mathrm{N}^{\mathrm{o}} 10484$ de Chota. Se identificó el nivel de pensamiento crítico y creativo alcanzado por los estudiantes del sexto grado, después de haber participado en la ejecución del Programa 
de Experiencias de Aprendizaje, utilizando la plataforma virtual Moodle. Finalmente, mediante los resultados obtenidos en datos estadísticos se estableció que, el nivel de pensamiento crítico y creativo de los estudiantes de sexto grado de la Institución educativa $\mathrm{N}^{\mathrm{o}} 10484$ de Chota, ha desarrollado significativamente con la intervención del Programa de estrategias de aprendizaje utilizando como estrategia la plataforma virtual Moodle.

\section{PROPUESTA:}

Programa de Experiencias de Aprendizaje utilizando la Plataforma Virtual Moodle, como estrategia para desarrollar el pensamiento crítico y creativo en los estudiantes de la Institución Educativa $N^{o} 10384$ - Chota.

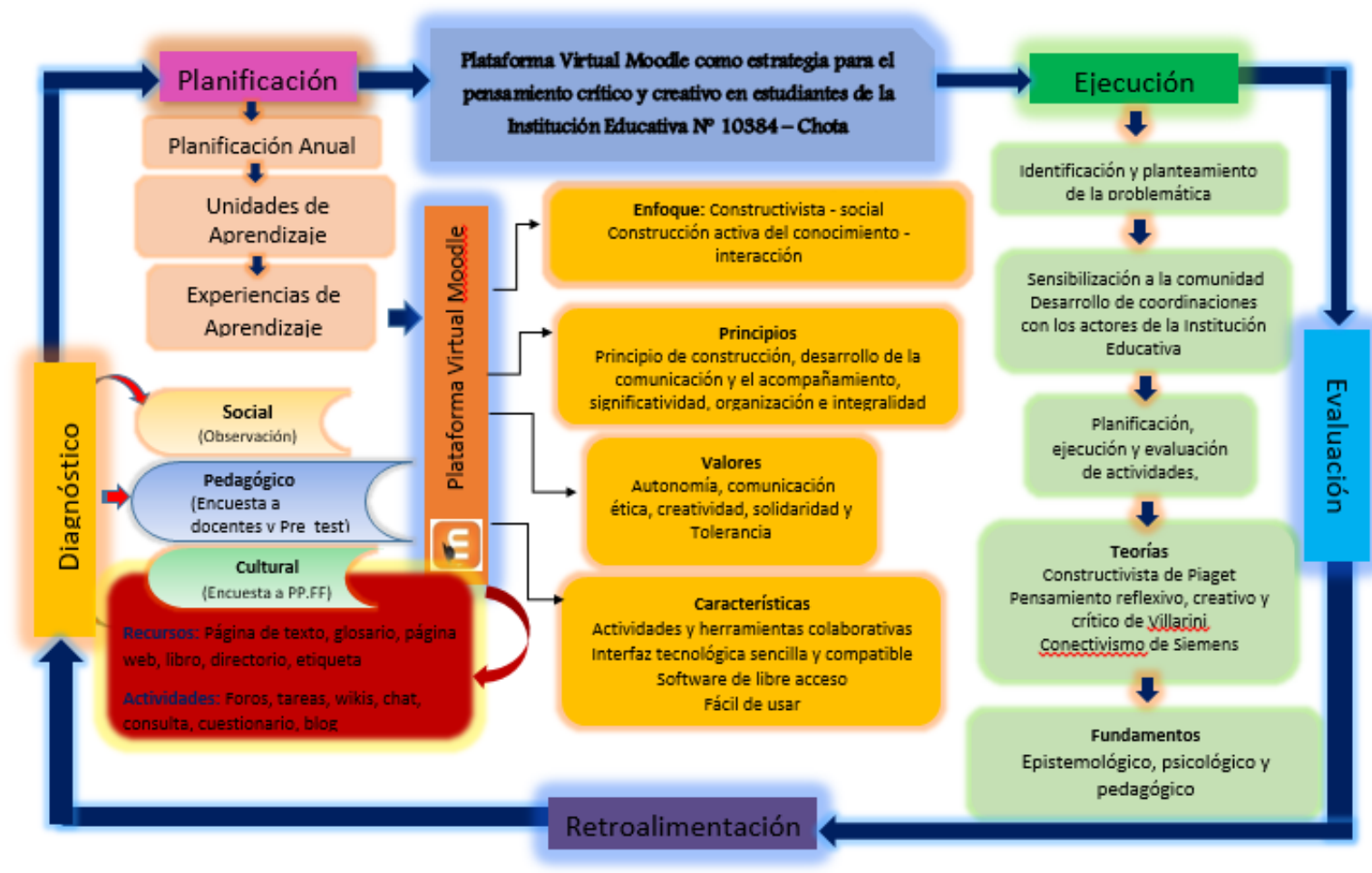

\section{REFERENCIAS BIBLIOGRAFICAS}

Arévalo, M. D., \& Nauta, H. M. (2012). LA TEORIA PIAGETANA. Obtenido de https://dspace.ucuenca.edu.ec/bitstream/123456789/2197/1/tps700.pdf

Bachillerato Internacional. (2020). La importancia del pensamiento crítico en el siglo XXI. Obtenido de [Entrada de blog]: https://blogs.ibo.org/blog/2020/01/14/laimportancia-del-pensamiento-critico-en-el-siglo-xxi/?lang=es

Congreso de la Repúlica Perú. (2018). Obtenido de https://www.congreso.gob.pe/congresistas2016/MoisesGuia/NotasPrensa/ 
De medio, C., Limongelli, C., Temperini, M., \& Sciarrone, F. (2019). MoodleREC: A recommendation system for creating courses using the moodle e-learning platform. (Computers in Human Behavior, Editor) doi:https://doi.org/10.1016/j.chb.2019.106168Get

Espinoza, M. Y., \& Zambrano, W. D. (2020). Los estilos de aprendizaje de estudiantes rurales en la implementación de una plataforma MOODLE. http://dx.doi.org/10.23857/dc.v6i5.1612

Facione, P. A. (2017). Pensamiento Crítico: ¿Qué es y por qué es importante? http://eduteka.icesi.edu.co/pdfdir/PensamientoCriticoFacione.pdf

Guzzetti, P. C. (2020). Plataforma virtual: una herramienta didáctica para el Proceso de. 4(2). doi:https://doi.org/10.37811/c1_rcm.v4i2.122

Hernández, R., Fernández, C., \& Baptista, M. P. (2014). Metodología de la Invetigación. https://www.uca.ac.cr/wp-content/uploads/2017/10/Investigacion.pdf

Herrera, P. V. (2020). Reflexiones sobre la Observación. https://wiki.ead.pucv.cl/images/d/db/T9_-

_Reflexiones_sobre_la_Observaci\%C3\%B3n_\%281\%29.pdf

Horn, S., \& Veermans, V. (2019). Critical thinking efficacy and transfer skills defend against 'fake news' at an international school in Finland. Obtenido de https://journals.sagepub.com/doi/full/10.1177/1475240919830003

Ikhsan, J., Handoyo, K., \& Nur, T. (2020). Fostering Student's Critical Thinking through a Virtual. https://online-journals.org/index.php/i-jim/article/view/13069

Muñiz, J., \& Fonseca-Pedrero, E. (2018). Diez pasos para la construcción de un test. Psicothema.

Robles, B. F. (2019). Población y muestra. http://200.62.226.189/PuebloContinente/article/view/1269/1099

Rus, E. (2020). Investigación Aplicada. https://economipedia.com/definiciones/investigacion-aplicada.html

Sirwan, S., Radhiani, A., \& Sartika, S. (2021). ). Development of Virtual Learning System Based On Moodle as A Platoform Online Learning th the Covid-19 Pandemic. Jurnal Basicedu, 5(5), $4314 \quad$ - $4327 . \quad$ doi:: https://doi.org/10.31004/basicedu.v5i5.1551 
Unger, M. M., \& BenDavid-Hadar, I. (2021). Does Home Schooling Improve Creative Thinking and Social Competencies among Children? Home Schooling in Israel. $\begin{array}{llllll}\text { Journal of } & \text { School } & \text { Choice, } & 1 & - & 28 .\end{array}$ doi:https://doi.org/10.1080/15582159.2021.1977584

Unidad de Gestión educativa Hualgayoc - Bambamarca. (2017). Proyecto Educativo Local de la provincia de Hualgayoc periodo 2017 - 2021. Obtenido de https://www.ugelbambamarca.gob.pe/jp_PEL/Proyecto_Educativo_Local.pdf

Velásquez , B. R., Salazar, M. R., Aldana, J. M., Morales, K. L., Castañeda, C. E., Noguera, K. C., . . . Villela, C. E. (2021). Teoría del aprendizaje conectivista, sobresaliente del siglo XXI. https://revistacunori.com/index.php/cunori/article/view/159/216

Villarini, Á. R. (2019). Un modelo del pensamiento reflexivo, creativo y crítico como competencia humana Obtenido de https://www.magisterio.com.co/articulo/un-modelo-del-pensamiento-reflexivocreativo-y-critico-como-competencia-humana-general

Wojciehowski, M., \& Ernst, J. (2018). Creative by Nature: Investigating the Impact of Nature Preschools on Young Children's Creative Thinking. International Journal of Early Childhood Environmental Education, 6(1), 3-20. Obtenido de https://eric.ed.gov/?id=EJ1193490 